\title{
Tunable Hydrogels with Improved Viscoelastic Properties from Hybrid Polypeptides.
}

\author{
Dimitrios Skoulas ${ }^{1}$, Gaetano Mangiapia², Daniele Parisi ${ }^{3}$, Maria Kasimatis ${ }^{1}$, \\ Emmanouil Glynos ${ }^{3}$, Efstratios Stratikos ${ }^{1}$, Dimitris Vlassopoulos $^{3}$, Henrich \\ Frielinghaus ${ }^{4}$ and Hermis Iatrou ${ }^{1}$, * \\ 1. National and Kapodistrian University of Athens, Department of Chemistry, \\ Panepistimiopolis, Zografou, 15771, Athens, Greece \\ 2. German Engineering Material Science (GEMS) at Heinz Maier-Leibnitz Zentrum (MLZ), \\ Helmholtz-Zentrum Hereon, Lichtenbergstraße 1, 85748 Garching, Germany \\ 3. FORTH, Institute for Electronic Structure and Laser, Heraklion 71110, Greece and \\ Department of Materials Science \& Technology, University of Crete, Heraklion 71003, \\ Greece \\ 4. Jülich Centre for Neutron Science JCNS at Heinz Maier-Leibnitz Zentrum (MLZ), \\ Forschungszentrum Jülich GmbH, Lichtenbergstraße 1, 85748 Garching, Germany
}

\section{MATERIALS}

Boc-His $(\operatorname{Trt})-\mathrm{OH}(>99 \%)$ was purchased from Christof Senn Laboratories AG. Triphosgene (99\%) was acquired from Acros Organics. Thionyl chloride ( $>99 \%)$ and L-Glutamic acid $\gamma$-benzyl ester were purchased from Aldrich. $N^{\alpha}, N^{\varepsilon}$-di-(tertbutoxycarbonyl)-L-Lysine (>99 \%) was purchased from Alpha Aesar. Triethylamine ( $>99 \%$, Acros Organics) was dried over calcium hydride for one day and then distilled and stored over sodium. Then, the desired amount was distilled to another calibrated apparatus prior to use. $N, N$-Dimethylformamide (DMF) was supplied from Fischer Scientific (99.91\%, special grade for peptide synthesis with less than $50 \mathrm{ppm}$ active impurities) and was further purified by short-path fractional distillation under high vacuum. The middle fraction was always used. Trifluoroacetic acid ( $\geq 99.5 \%$, TFA) was purchased from Fischer Scientific. Thionyl chloride $\left(\mathrm{SOCl}_{2}\right)(99.7 \%$, distilled before use) was obtained from Acros Organics. Triethylsilane (99\%), limonene (97\%) and diethyl ether $(\geq 99 \%)$ were purchased from Aldrich. Purification of dichloromethane (anhydrous, $\geq 99.8 \%$, Sigma-Aldrich) and tetrahydrofuran (THF, max $0.005 \%$ water, Merck Millipore) was performed using standard high vacuum techniques reported elsewhere. ${ }^{1}$ Ethyl acetate purchased from Merck Millipore (>99.5\%) was fractionally distilled over phosphorous pentoxide. Hexane ( $>99 \%$, Merck Millipore) was fractionally distilled over sodium and subsequently distilled over $n$-BuLi. Acetonitrile (99.9\%) was purchased from Aldrich. Distilled water was further purified by means of a Milli-Q water purification system (18.2 $\mathrm{M} \Omega \mathrm{cm}$, Merck Millipore). Thiophene-free benzene was treated with calcium hydride, which allowed to be stirred overnight to react with moisture. Then, it was distilled under high vacuum in a nearby flask containing normal butyl lithium, which reacted with any impurities. End-functionalized 
bis(amine)-poly(ethylene oxide) $\left(\mathrm{H}_{2} \mathrm{~N}-\mathrm{PEO}-\mathrm{NH}_{2}\right)$ of varying molecular weights (5.95 $\mathrm{x} 10^{3}, 10.0 \times 10^{3}$ and $20.0 \times 10^{3} \mathrm{~g} / \mathrm{mol}$ ) was purchased from Aldrich and was employed as a difunctional macroinitiator. The enzymes Leucine Aminopeptidase (LAP) and Trypsin were purchased from Aldrich. Fluorescamine was purchased from Alpha Aesar.

\section{SYNTHESIS OF MONOMERS}

Synthesis of $\varepsilon$-tert-butyloxycarbonyl-L-Lysine N-carboxy anhydride (N\&-BOC-LLYS NCA): $\varepsilon$-tert-Butyloxycarbonyl-L-lysine-NCA was synthesized according to a previously reported method [2]. Briefly, 46.37g $\left(0.134\right.$ moles) of $\mathrm{N}^{\alpha}, \mathrm{N}^{\varepsilon}$-Di-(tertbutoxycarbonyl)-L-lysine was added into a flask, which was attached to the vacuum line and pumped overnight. Then, $1000 \mathrm{~mL}$ of purified ethyl acetate was distilled, followed by argon insertion and addition of $14.6 \mathrm{~g}(0.049 \mathrm{~mol})$ triphosgene. The mixture was left to react for 10 minutes. Then, triethylamine $(18.6 \mathrm{~mL}=0.134 \mathrm{~mol})$ diluted in dry ethyl acetate was added dropwise, and the solution was immersed in an ice-water bath for 6 hours. The precipitate was filtered, in order to remove the $\mathrm{HCl}$ salt of triethylamine. Afterward, the solution of the reaction was extracted firstly with an aqueous solution of $\mathrm{NaHCO}_{3}$ and then with Milli-Q water, until neutral $\mathrm{pH}$ of the aqueous phase was achieved. After extraction, the organic phase containing the purified NCA was recrystallized three times from ethyl acetate/hexane $(1 / 5 \mathrm{v} / \mathrm{v})$ under high vacuum in a custom-made apparatus. The yield was $60 \%$ (27.8 gr). The purity was confirmed by ${ }^{1} \mathrm{H}$ NMR along with FTIR spectroscopy.<smiles>CCNC(=O)OC(C)(C)C</smiles><smiles></smiles>

Figure S1: Synthesis of Ne-Boc-L-Lys NCA L-NCA 


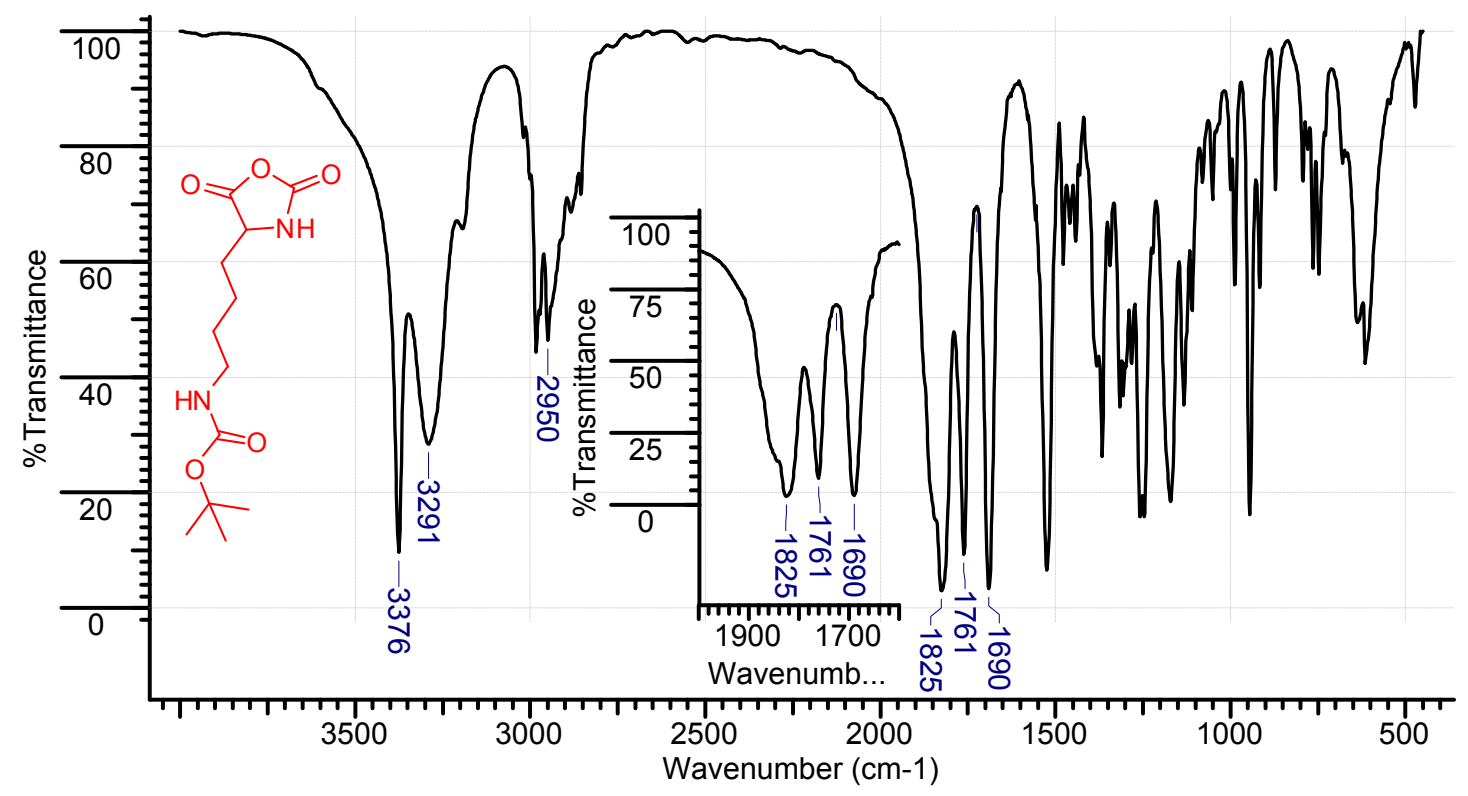

Figure S2: IR spectrum of Ne-Boc-L-Lys NCA

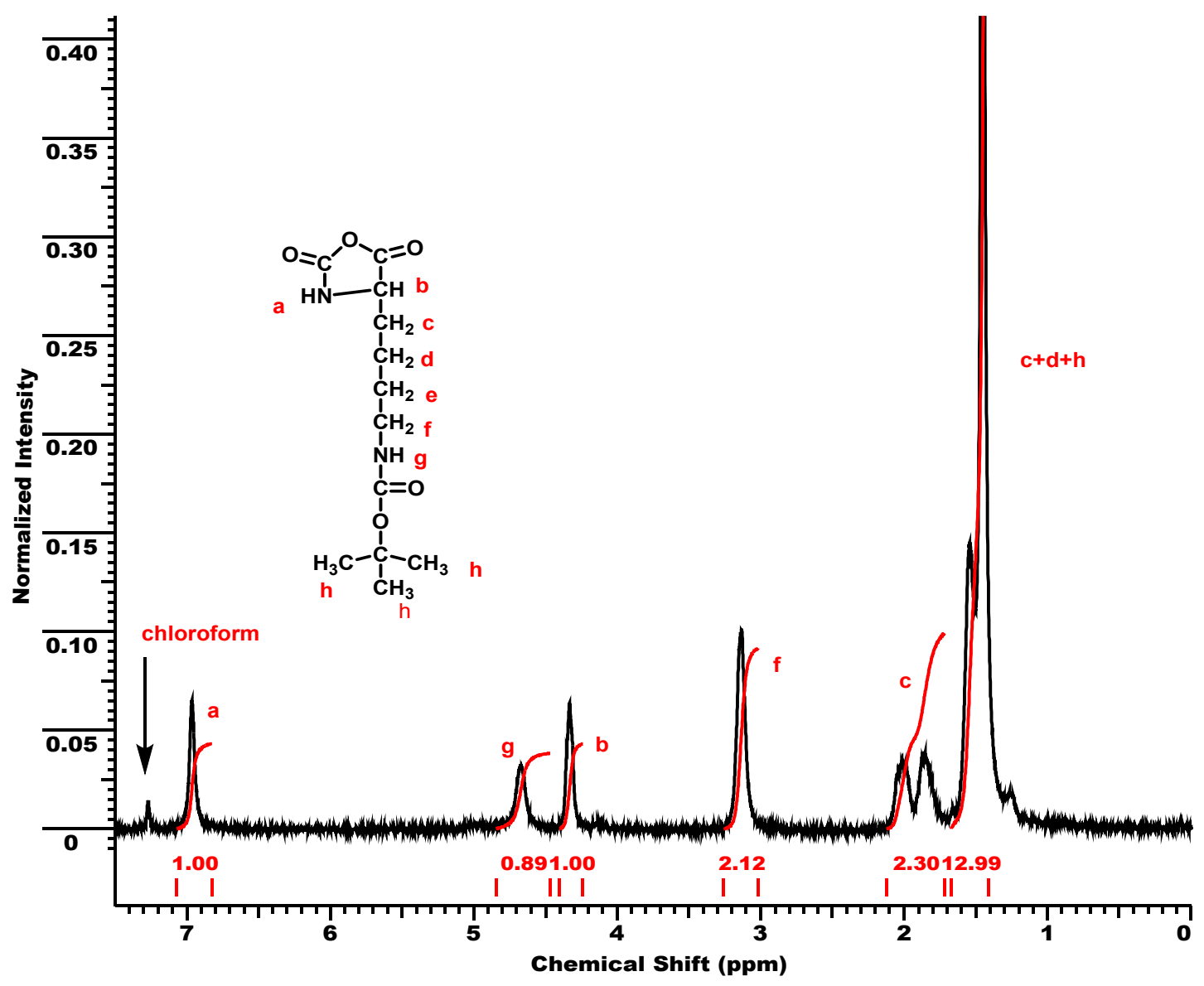

Figure S3: NMR spectrum of $N \varepsilon-B o c-L-L y s N C A$ 
Synthesis of $\gamma$-benzyl-L-glutamate N-carboxy anhydride (BLG-NCA): The NCA of $\gamma$-benzyl-L-glutamate was synthesized according to a previously reported method [3]. Briefly, 29.4g $(0.122 \mathrm{~mol})$ of $\gamma$-benzyl-L-glutamate was suspended in dry THF followed by the addition of limonene $(21,8 \mathrm{~mL})$ and triphosgene $13.3 \mathrm{~g}(0.045 \mathrm{~mol})$. The mixture was heated at $70^{\circ} \mathrm{C}$ until the solution became clear, indicating the formation of the NCA. The solvent was distilled off in the vacuum line, and fresh dry ethyl acetate was distilled in the flask to dissolve the crude NCA. Then, the solvent was removed again by distillation. This procedure was repeated twice in order to remove the excess of phosgene that sublimes under high vacuum. The unreacted species, such as free amino acids along with the $\mathrm{HCl}$ salts of the amino acids produced during the synthesis, were removed by extraction with an alkali solution in water. The resulted BLG-NCA was further purified by three recrystallizations from dry ethyl acetate/hexane $(1 / 5 \mathrm{v} / \mathrm{v})$ under high vacuum at $-20^{\circ} \mathrm{C}$. The yield of BLG-NCA was $65 \%$ (19.4 g). The purity of BLG-NCA was confirmed by ${ }^{1} \mathrm{H}$ NMR and FTIR spectroscopy

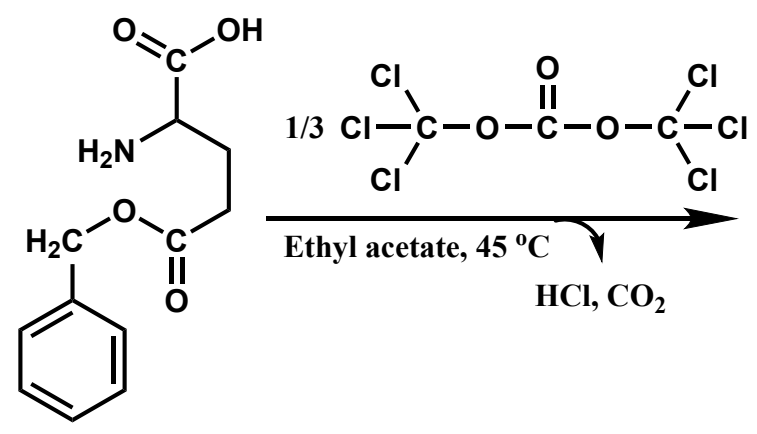<smiles>O=C(CCC1[NH2+]C(=O)OC1=O)OCc1ccccc1</smiles>

Figure S4: Synthesis of BLG-NCA 


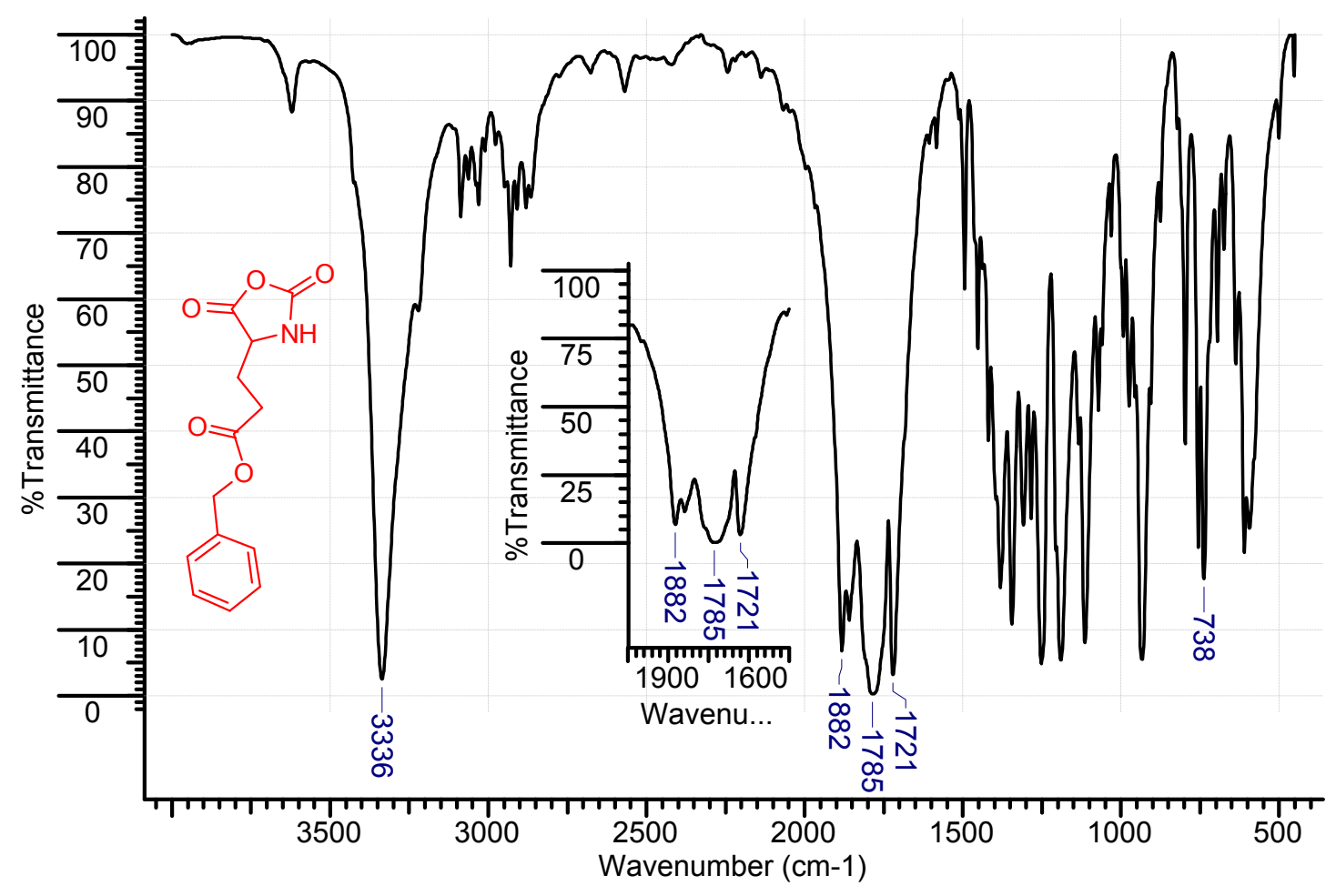

Figure S5: IR spectrum of BLG-NCA

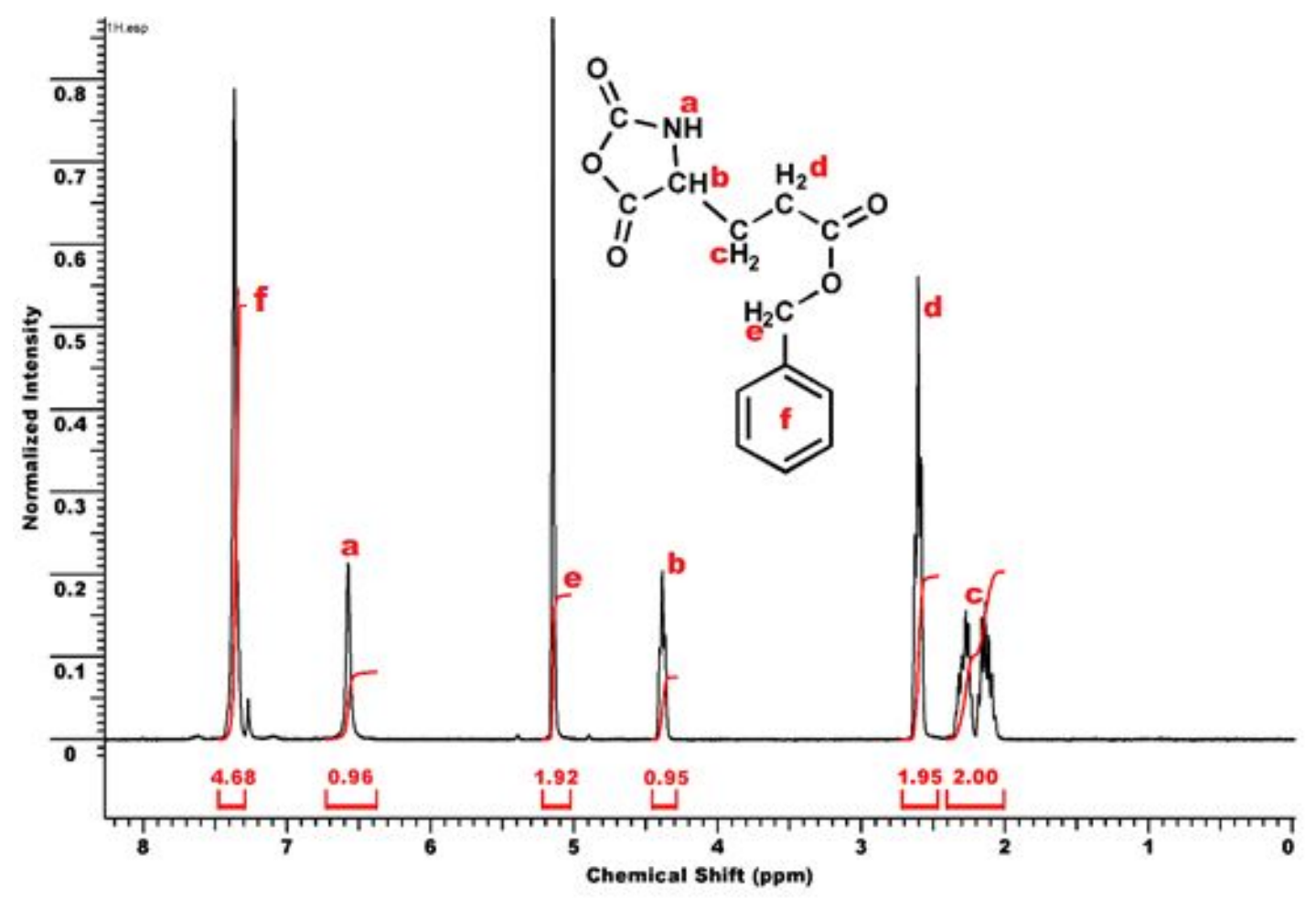

Figure S5: NMR spectrum of BLG-NCA 
Synthesis of $\mathrm{N}^{\mathrm{im}}$ trityl protected $\mathrm{N}$-carboxy anhydride of L-histidine (Trt-HISNCA). The synthesis of Trt-HIS-NCA was reported elsewhere. ${ }^{4}$ In a $500 \mathrm{~mL}$ round bottom flask $20 \mathrm{~g}(40.2 \mathrm{mmol})$ of Boc-His(Trt)-OH was added and dried overnight under high vacuum. Then $150 \mathrm{~mL}$ of THF were distilled in the flask, giving a clear yellowish solution. The reaction flask was placed in an ice-bath, filled with Argon and $3.25 \mathrm{~mL}$ (44.2mmol) of thionyl chloride diluted in $20 \mathrm{~mL}$ of THF were added dropwise in a period of 10 minutes. After 2 hours, the solution was poured in $2 \mathrm{~L}$ of cold $(\mathrm{Et})_{2} \mathrm{O}$ with precipitation of Trt-His-NCA $\cdot \mathrm{HCl}$ as the major product. Finally, the solid was filtered (glass sintered filter 3) and then transferred to a round bottom flask of $500 \mathrm{~mL}$ and dried in $\mathrm{HV}$ giving $17.2 \mathrm{~g}$. Recrystallization was conducted to the above solid mixture, containing the $\mathrm{HCl}$ salt, free anhydride and the initial substrate by distilling $300 \mathrm{~mL}$ of ethyl acetate under HV. The round bottom flask of $500 \mathrm{ml}$, containing the suspension, was removed from $\mathrm{HV}$ and placed into a water bath, at $45^{\circ} \mathrm{C}$ for 1 hour, resulting in dissolution. Then the solution was cooled to $0^{\circ} \mathrm{C}$ with an ice bath and Trt-His NCA. $\mathrm{HCl}$ was formed as a precipitate which was isolated as the only product after filtration. The NCA salt was transferred to another flask of $500 \mathrm{ml}$ and dried overnight under HV $(12.5 \mathrm{~g}=28 \mathrm{mmol})$. Subsequently, $200 \mathrm{~mL}$ of EtAc was distilled into the flask, the flask was removed from $\mathrm{HV}$, filled with Ar and placed in an ice bath. At $0^{\circ} \mathrm{C} 3.5 \mathrm{ml}(28 \mathrm{mmol})$ of the stoichiometric amount of triethylamine dissolved in $50 \mathrm{ml}$ of the same solvent was slowly added dropwise under vigorous stirring (duration of addition 1 hour). The resulted triethylamine hydrochloride was filtered off and the filtrate was poured into 1.5 L of non-solvent hexane in order to recrystallize the Trt-His-NCA. The second recrystallization occurred with a mixture of solvent/non-solvent EtAc/Hexane (1:5) and the white solid precipitate Trt-His-NCA was isolated by filtration. Finally, Trt-HisNCA was dried under high vacuum overnight and transferred into the glove box resulting in $11.05 \mathrm{~g}(27 \mathrm{mmol}, 67 \%$ yield). The purity of Trt-HIS-NCA.HCl was confirmed by ${ }^{1} \mathrm{H}$ NMR and IR spectroscopy
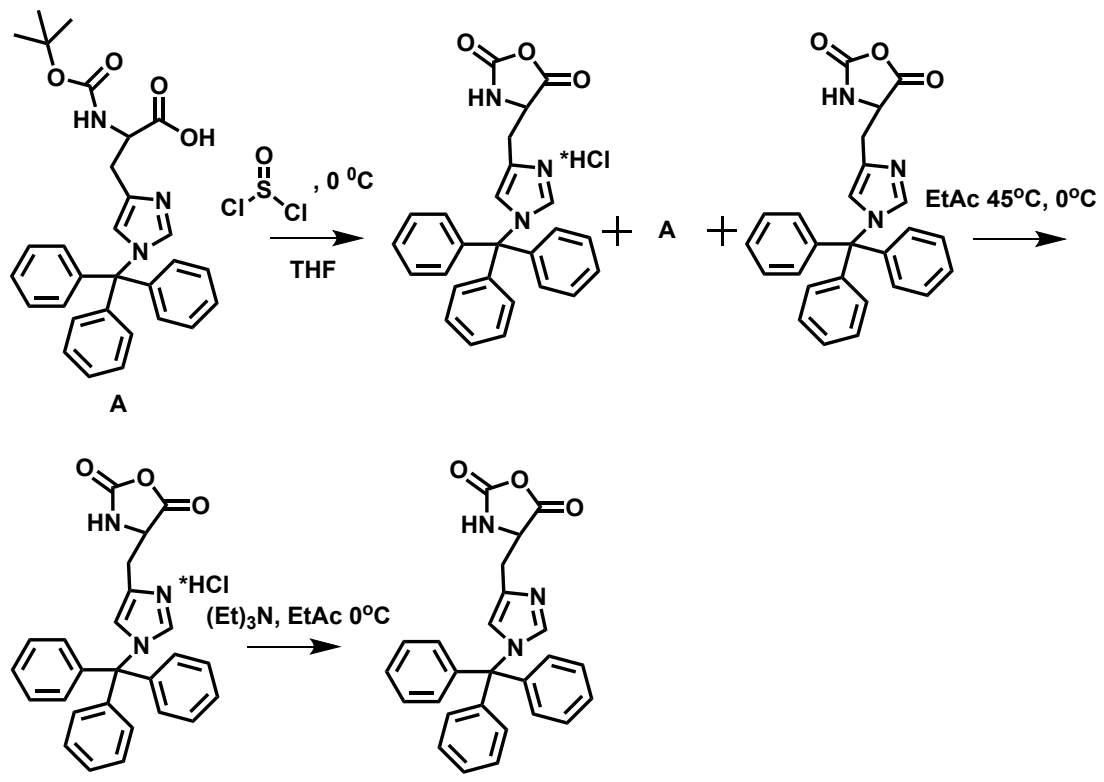

Figure S6: Synthesis of Trt-HIS-NCA 


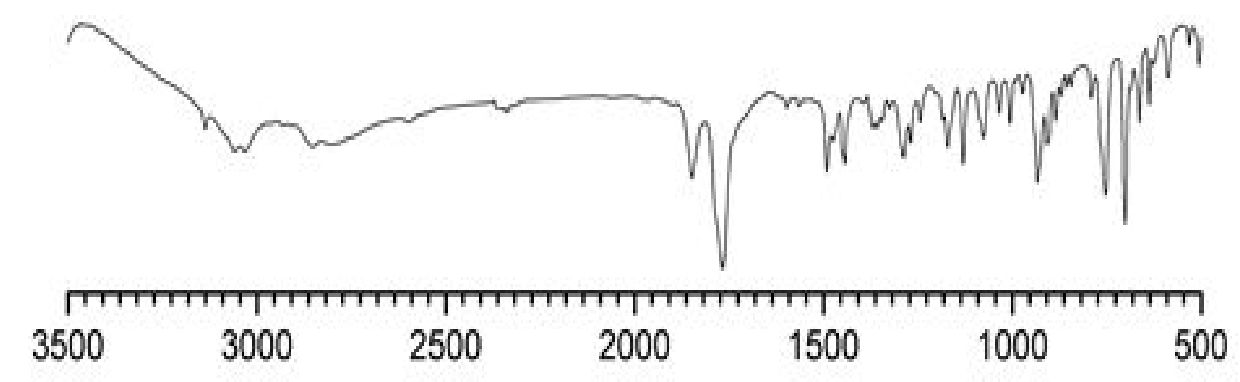

Figure S7: IR spectrum of Trt-HIS-NCA

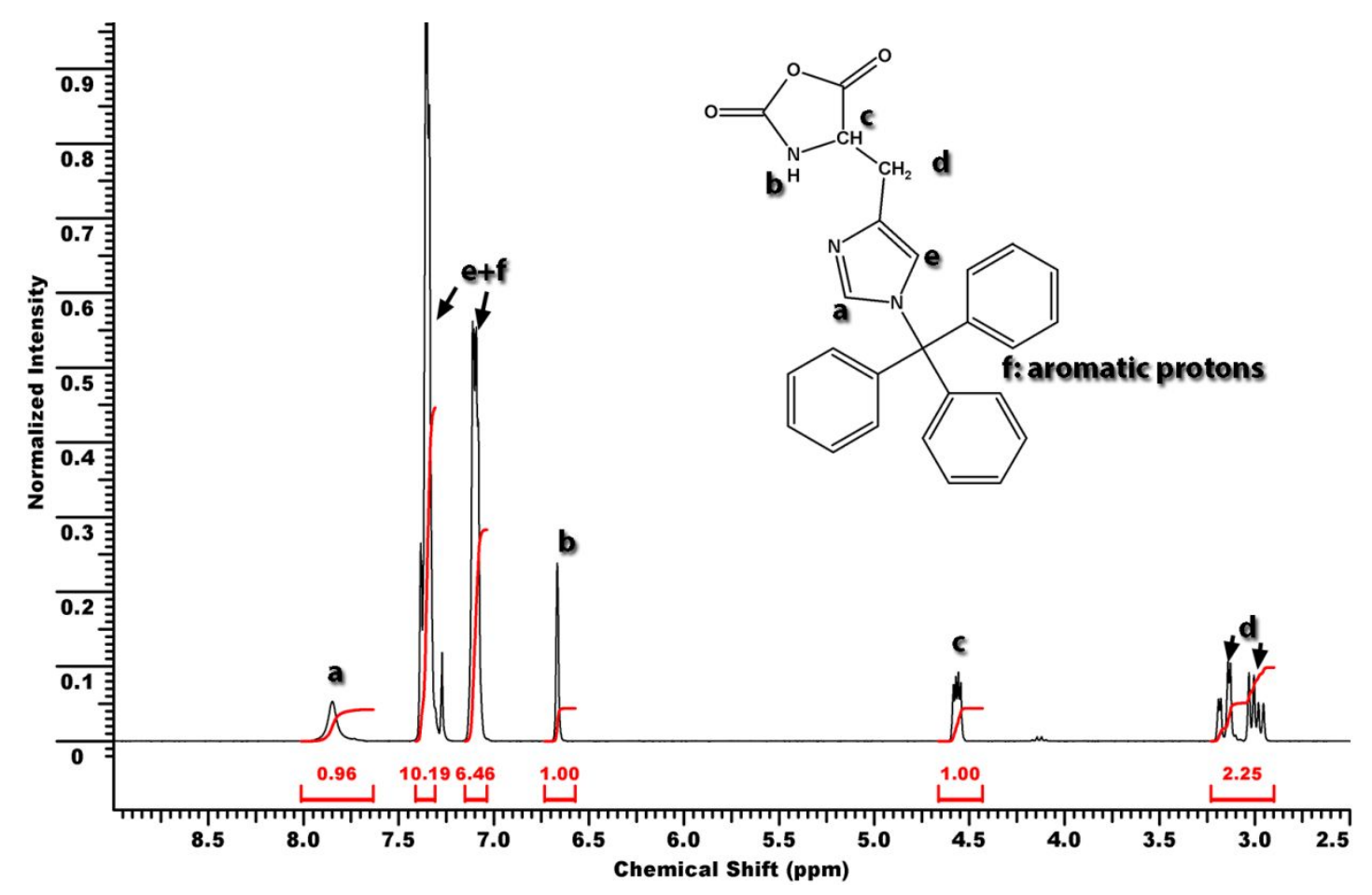

Figure S8: NMR spectrum of Trt-HIS-NCA

\section{POLYMER SYNTHESIS}

All manipulations and synthetic procedures were performed under high vacuum in custom-made glass reactors, equipped with break-seals, high-vacuum stopcock, glasscovered magnets and constrictions for the addition of reagents under the guidelines of the high-vacuum techniques. ${ }^{1}$ Each apparatus features two custom-made glass ampules with two ground joints for each of the ampules, for addition of the monomers.

The general procedure for the synthesis of PLys- $b$-(PHis-co-PBLG)-PEO- $b$-(PHis-coPBLG)- $b$-PLys with $\mathrm{M}_{\mathrm{n}}$ of PEO $20.0 \times 10^{3} \mathrm{~g} / \mathrm{mol}, 80$ monomeric units of L-histidine, 80 monomeric units of $\gamma$-benzyl-L-glutamate and 240 monomeric units of L-Lysine will 
be presented below. All hybrid-polypeptides were synthesized following the same procedure, with alteration only in the amount of the monomers used in order to reach the molecular weights.

The glass vessel was initially attached to the vacuum line through the ground joint, was evacuated and was flame-dried several times. Then, it was transferred to the glove box and $0.2 \mathrm{~g}$ of end-functionalized bis(amine)-poly(ethylene oxide) with $\mathrm{M}_{\mathrm{n}}=20.0 \times 10^{3}$ $\mathrm{g} / \mathrm{mol}\left(1.0 \times 10^{-5} \mathrm{~mol}\right)$ were added. Subsequently, $30 \mathrm{~mL}$ of highly dry benzene were distilled and the macroinitiator was dissolved. The solution was stirred for two hours and benzene was distilled off to dryness. The apparatus was left overnight to the high vacuum line. The next day, $30 \mathrm{~mL}$ of highly pure DMF were distilled, followed by the dissolution of the macroinitiator. Then, the apparatus was inserted again in the glove box, a mixture of $0.338 \mathrm{~g}$ of His-NCA $\left(8.0 \times 10^{-4} \mathrm{~mol}\right)$ and $0.210 \mathrm{~g}$ of BLG-NCA $(8.0 \mathrm{x}$ $10^{-4} \mathrm{~mol}$ ) was added in one-side ampoule, which was equipped with a ground joint and a constriction.

The apparatus was attached to the vacuum line through the ground joint of the ampoule containing the NCAs, it was evacuated and approximately $5.0 \mathrm{~mL}$ of highly pure DMF were distilled. It was removed from the vacuum line through heat-sealing a constriction, and the mixture of both monomers was dissolved. The solution of the NCAs was added through the rupture of the break-seal of the ampoule, and the solution was vigorously stirred. The consumption of the monomers was monitored by FT-IR through the removal of an aliquot of the solution in the glove box. Periodically, the solution was pumped to remove the $\mathrm{CO}_{2}$ produced during the polymerization. The polymerization lasted 7 days.

Subsequently, the monomer $\varepsilon$-tert-butyloxycarbonyl-L-Lysine N-carboxy anhydride was added, using the other ampule and following exactly the same procedure. We added $0.653 \mathrm{~g}$ of the Lys-NCA $\left(2.4 \times 10^{-3} \mathrm{~mol}\right)$. The polymerization lasted 2 days. After completion, the polymer was precipitated in diethyl ether $(\sim 300 \mathrm{~mL})$ and filtered using a Buchner device with a $0.45 \mu \mathrm{m}$ filter. Then, it was dried under high vacuum $(0.82 \mathrm{~g}$ $77 \%$ yield).

The polymer was suspended in $\mathrm{CH}_{2} \mathrm{Cl}_{2}(10.0 \mathrm{~mL})$ and an equal volume of TFA was added. It was completely dissolved and left to be deprotected for 1 hour at room temperature. Then, an amount of triethyl silane was added. The solution was distilled in the vacuum line to remove all solvents. The remaining undissolved solid was suspended in water and dialyzed twice against 2 liters of Milli-Q water with $\mathrm{pH} \sim 3$ (adjusted with a dilute aqueous solution of $\mathrm{HCl}$ ), twice in Milli-Q water with $\mathrm{pH} \sim 7.4$ until constant $\mathrm{pH}$ (adjusted with a dilute aqueous solution of $\mathrm{NaOH}$ ) and twice in pure Milli-Q water. Finally, the aqueous polymer solution was freeze-dried to yield the final polymer, which had a mass of $0.50 \mathrm{~g}$ (46\% yield).

\section{METHODS AND INSTRUMENTATION}

Sample preparation. The hydrogels were prepared by the simple addition of Milli-Q water to the polymer. For all measurements, tests and experiments the concentration was always $5 \% \mathrm{w} / \mathrm{w}$ or 1:20 w/w (polymer:water w/w). For SANS experiments, water 
was replaced by $\mathrm{D}_{2} \mathrm{O}$ (Armar chemicals, Döttingen, Switzerland. 99.8\%) in order to minimize the incoherent scattering contribution to the measured cross sections.

Size-exclusion chromatography. Size-exclusion chromatography (SEC) was used to determine the $M_{n}$ and $M_{w} / M_{n}$ values. The analysis was performed using a SEC equipment. The system was composed of a Waters 600 high-pressure liquid chromatographic pump, Waters Ultrastyragel columns (HR-2, HR-4, HR-5E and HR6E), a Waters 410 differential refractometer detector and a Precision PD 2020 twoangles $\left(15^{\circ}, 90^{\circ}\right)$ light scattering detector at $60^{\circ} \mathrm{C}$. A $0.1 \mathrm{~N} \mathrm{LiBr} \mathrm{DMF} \mathrm{solution} \mathrm{was} \mathrm{used}$ as an eluent at a rate of $1 \mathrm{~mL} / \mathrm{min}$.

NMR spectroscopy. Proton nuclear magnetic resonance ( ${ }^{1} \mathrm{H}$ NMR) spectroscopy (300 $\mathrm{MHz}$ ) was performed using a Bruker $400 \mathrm{MHz}$ spectrometer in deuterated chloroform in order to confirm the successful synthesis of every monomer (NCA).FTIR. Fourier transform infrared (FTIR) spectroscopy measurements were performed with a Perkin Elmer Spectrum One instrument, in $\mathrm{KBr}$ pellets at room temperature, in the wavenumber range $450-4000 \mathrm{~cm}^{-1}$.

Circular Dichroism. Circular Dichroism was performed with a Jasco J-815 model, featuring a Peltier model PTC-423S/15 thermo stabilizing system. $1 \mathrm{~mm}$ Quartz Suprasil cell was used. Typical concentrations were about $5 \times 10^{-4} \mathrm{~g} / \mathrm{mL}$.

Rheology. The hydrogels were characterized by means of linear viscoelasticity. Dynamic oscillatory measurements using a strain-controlled ARES rheometer (from TA, USA) with a sensitive force rebalance transducer (100FRTN1). They included a) Dynamic frequency sweeps (DFS) with an imposed sinusoidal strain $\gamma=\gamma_{0} \sin \omega t$, having frequency $\omega$ and a small amplitude $\gamma_{0}$, in order to probe the linear viscoelastic spectrum, i.e., the frequency-dependent storage $\left(G^{\prime}\right)$ and loss $\left(G^{\prime \prime}\right)$ moduli in the oscillatory frequency range $0.01-100 \mathrm{rad} / \mathrm{s}$. The stress response was $\sigma=\sigma_{0}$ $\sin (\omega t+\delta)=\gamma_{0}\left(G^{\prime} \sin \omega t+G^{\prime \prime} \cos \omega t\right)$ with $\sigma_{0}$ being the stress amplitude and $\delta$ the phase angle. It is convenient to examine the frequency response in terms of loss factor, defined as $\tan (\delta)=G^{\prime \prime} / G^{\prime}$. The latter provides the inverse degree of elasticity of the system; the lower its value the larger the elastic contribution over the viscous one. $b$ ) Dynamic strain amplitude sweeps (DSS) at constant frequency (typically $1 \mathrm{rad} / \mathrm{s}$ ) and increasing strain amplitude, in order to determine the limits of linear viscoelasticity and at the same time explore the nonlinear response. c) Dynamic time sweeps (DTS) at a constant frequency (typically $1 \mathrm{rad} / \mathrm{s}$ ) and a small strain amplitude (in the linear viscoelastic regime), with duration long enough to ensure that steady state was reached. In order to erase the mechanical history of the sample and ensure reproducibility of the results, we pre-sheared the loaded samples by means of a DTS at large strain amplitude in the nonlinear regime (sample rejuvenation) and a subsequent DTS in the linear viscoelastic regime, to monitor the evolution of the shear-melted structure over time, until steady state (aging) was attained. To minimize the risk of solvent evaporation, the sample was sealed with a non-volatile immiscible low-viscosity fluid (silicon oil with viscosity $5 \mathrm{mPas}$ ) by means of a home-made trap (comprising a cylindrical ring surrounding the measuring system) while the entire fixture and ring with sealing fluid were isolated from the external environment by means of a cover (allowing only the rotation of the motor and connected tool) to avoid tiny effects of air current. 
Temperature control was achieved by means of a water/ethylene glycol recirculating bath. Different stainless steel cone-plate geometries were used depending on the sample's response and amount available: diameter of $8 \mathrm{~mm}$ and $0.166 \mathrm{rad}$ cone angle for HG1, 25mm and $0.1 \mathrm{rad}$ for HG6. Cross-hatched stainless steel parallel plates of 8 $\mathrm{mm}$ diameter were used for the rest of the samples to reduce the risk of wall slip.

Fluorescence measurements. All fluorescence measurements were performed with a TECAN SPARK 10M multimode microplate reader using 96-well black microplates (Greiner). To measure the enzymatic degradation of the hydrogel by either Trypsin or Leucine Aminopeptidase, $1.6 \mathrm{mg}$ of hydrogel was mixed with $0.1 \mathrm{nM}$ Trypsin or $100 \mathrm{nM}$ LAP in PBS and incubated at $37^{\circ} \mathrm{C}$ for $1 \mathrm{hr}$ or $24 \mathrm{hrs}$, respectively. At the end of the incubation process, a sample of $10 \mu \mathrm{L}$ from the reaction was mixed with $90 \mu \mathrm{L}$ of a solution of fluorescamine containing $1 \mathrm{mg} / \mathrm{mL}$ fluorescamine in acetonitrile, $15 \mu \mathrm{L}$ of $0.1 \mathrm{M}$ borate buffer at $\mathrm{pH} 8.0$ and $145 \mu \mathrm{L}$ of milli-Q water. After $5 \mathrm{~min}$, the fluorescence was measured using excitation at $405 \mathrm{~nm}$ and emission at $485 \mathrm{~nm}$.

SEM. The morphology of the hydrogels was probed with a field emission scanning electron microscope (FE-SEM, JEOL JSM-7000F). The measurements were conducted at FORTH, Institute for Electronic Structure and Laser.

Small-Angle Neutron Scattering. Small-Angle Neutron Scattering (SANS) measurements were performed at the KWS-1 diffractometer installed at the MaierLeibnitz Center in Garching, Germany. ${ }^{5}$ Neutrons with an average wavelength of $\lambda=$ $5.0 \AA$ and a wavelength resolution of $\Delta \lambda / \lambda \leq 0.10$ were used, by means of a mechanical velocity selector. A two-dimensional $128 \times 128$ pixel-based array scintillation detector set at three different collimation (C)/sample-to-detector (D) distances (namely $\mathrm{C}_{8} \mathrm{D}_{1.5}$, $\mathrm{C}_{8} \mathrm{D}_{8}$, and $\mathrm{C}_{20} \mathrm{D}_{20}$, with all distances in meters) measured neutrons scattered from the samples. These configurations allowed collection of data in the scattering vector modulus $(Q=4 \pi / \lambda \sin (\theta / 2))$ range between $2.02 \cdot 10^{-3}$ and $0.447 \AA^{-1}, \theta$ being the scattering angle. The investigated hydrogels were hosted in a sandwich cell using two quartz glasses distanced with a $2 \mathrm{~mm}$ spacer, which gave a good balance between illuminated volume and neutron transmission. The glasses were lodged in a holder and installed in a Peltier-based thermostat to keep the temperature constant. The samples were measured for sufficiently long time to have at least 2 million counts of neutrons. The obtained raw data were corrected for sample transmission, background- and empty cell scattering, and then radially averaged. Detector efficiency correction and transformation to absolute scattering cross sections were obtained by means of a secondary plexiglass standard. ${ }^{6}$ 


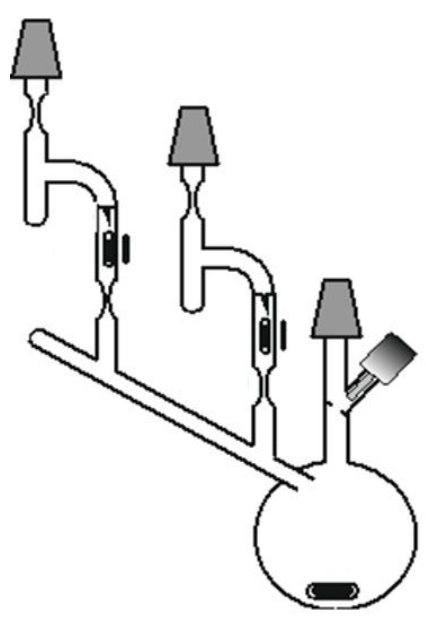

Figure S9: Custom-made glass reactors for the synthesis of PLys- $b$-(PHis-co-PBLG)PEO- $b$-(PHis-co-PBLG)- $b$-PLys

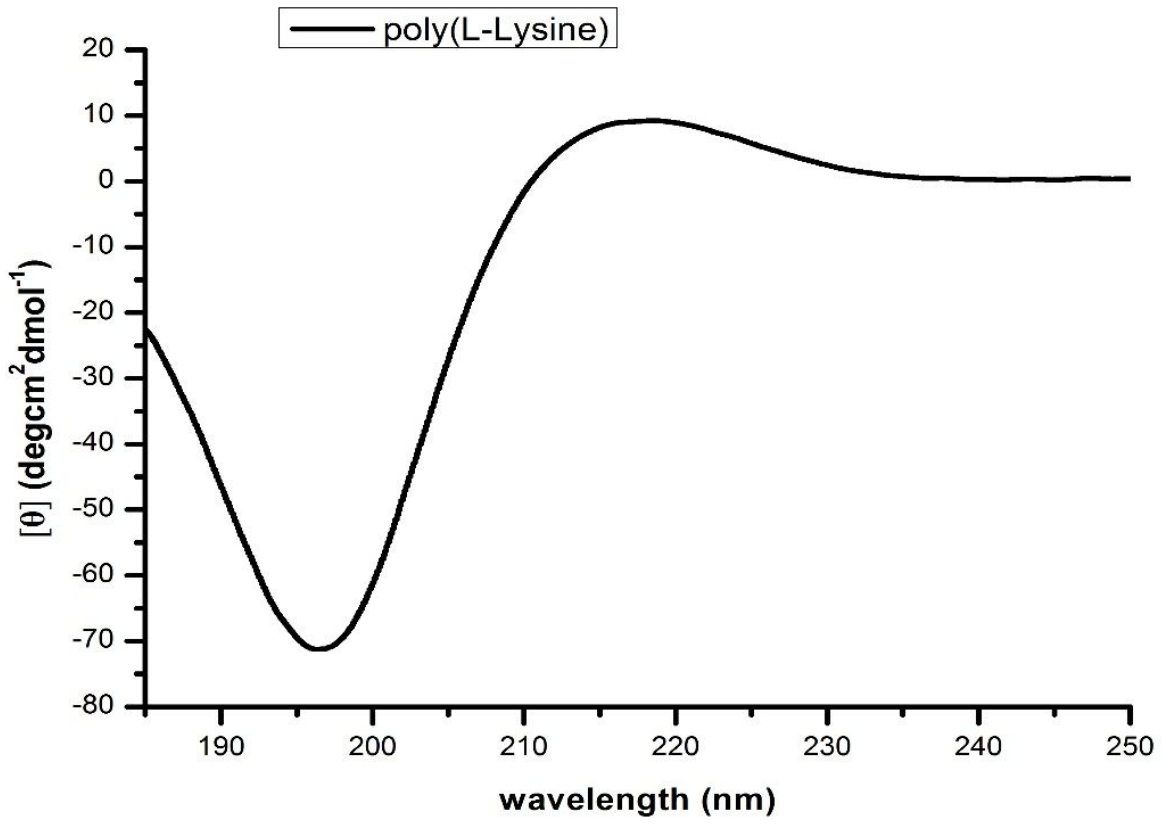

Figure S10: Circular dichroism spectrum of poly(L-Lysine) 

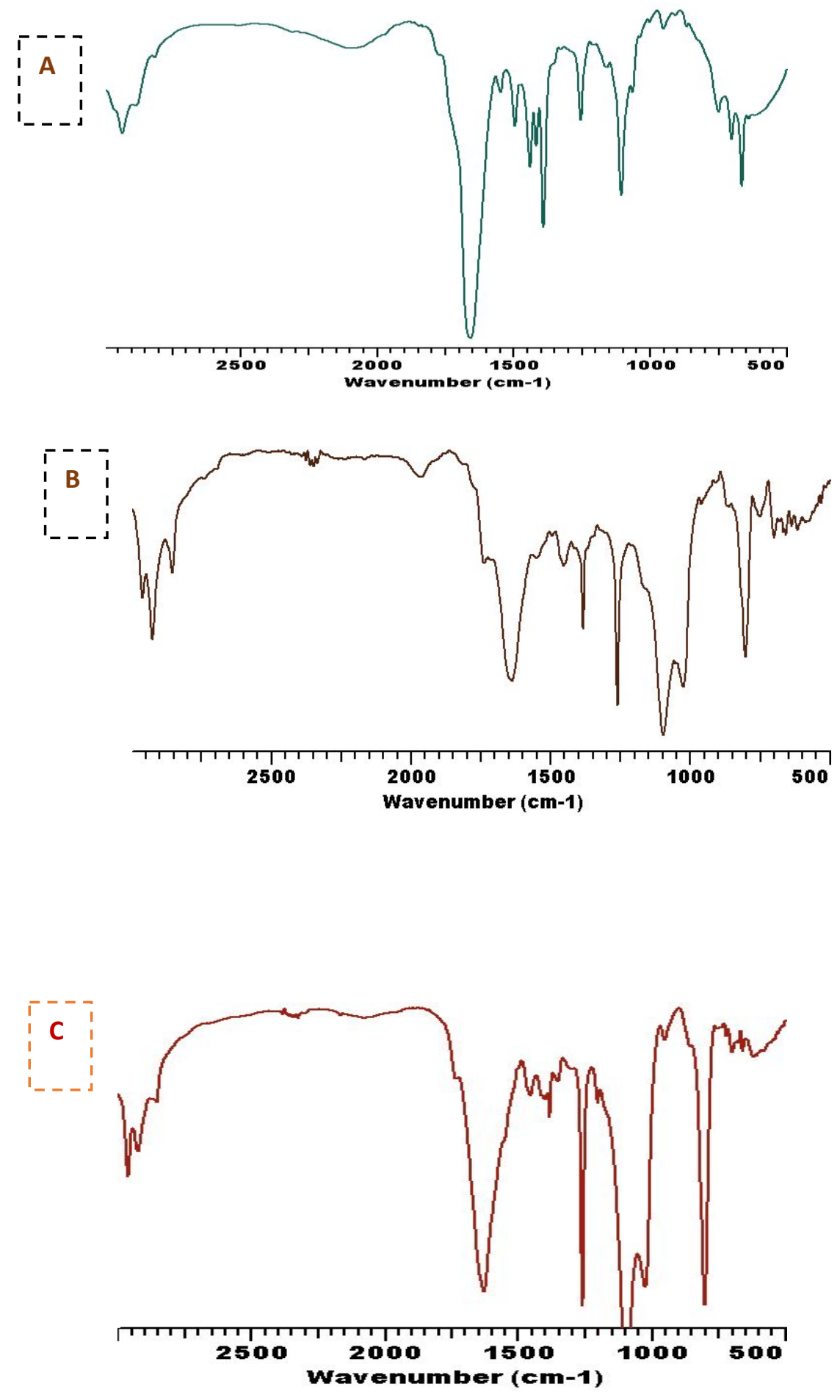

Figure S11: FT-IR spectra of PLys- $b$-(PHis- $c o-\mathrm{PBLG})-b$-PEO- $b$-(PHis- $c o-\mathrm{PBLG})-b-$ PLys A) After the copolymerization of PHis and PBLG B) After the polymerization of PLys C) After deprotection 


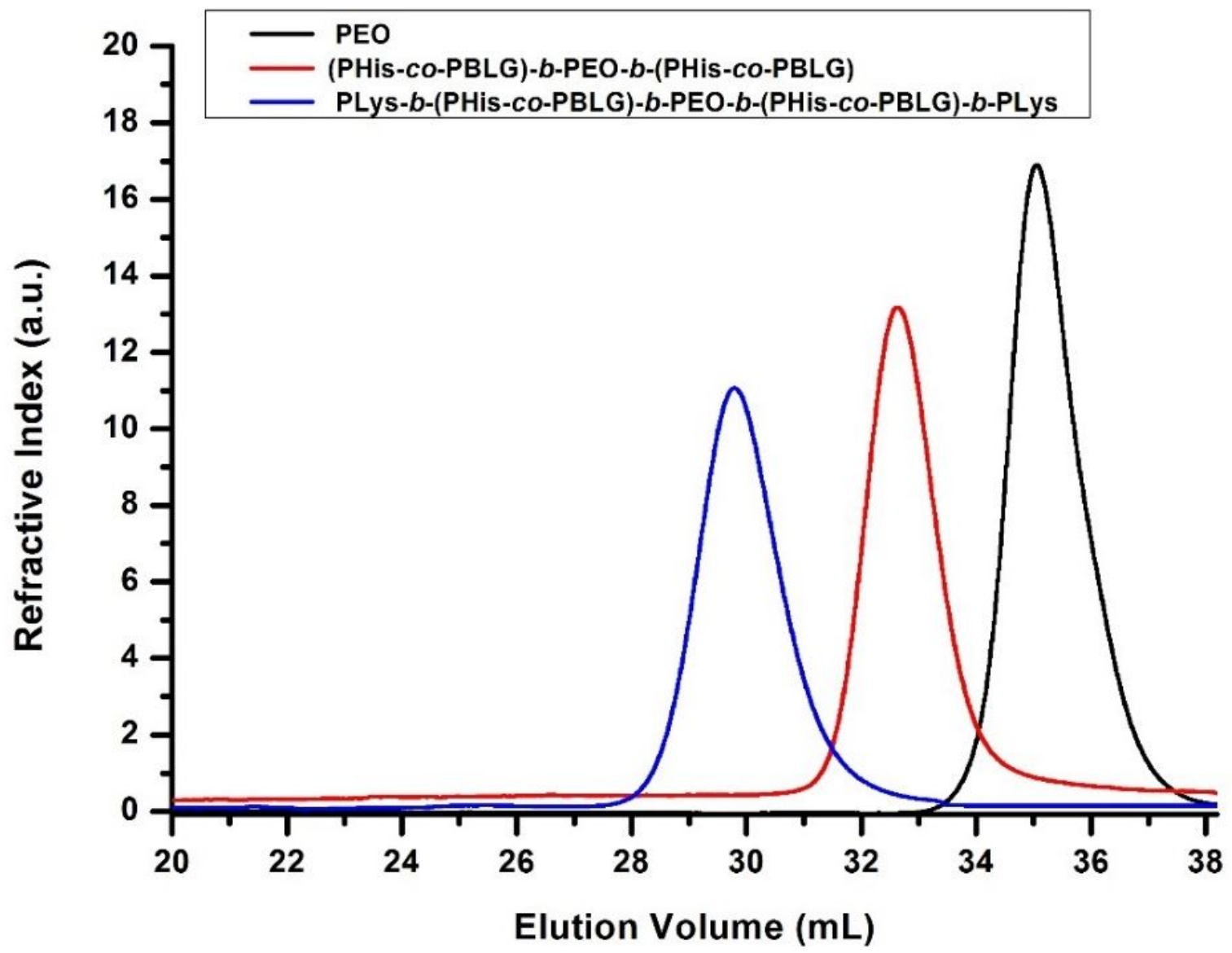

Figure S12: SEC eluograms of PLys-b-(PHis-co-PBLG)-b-PEO-b-(PHis-co-PBLG)b-PLys prior deprotection.

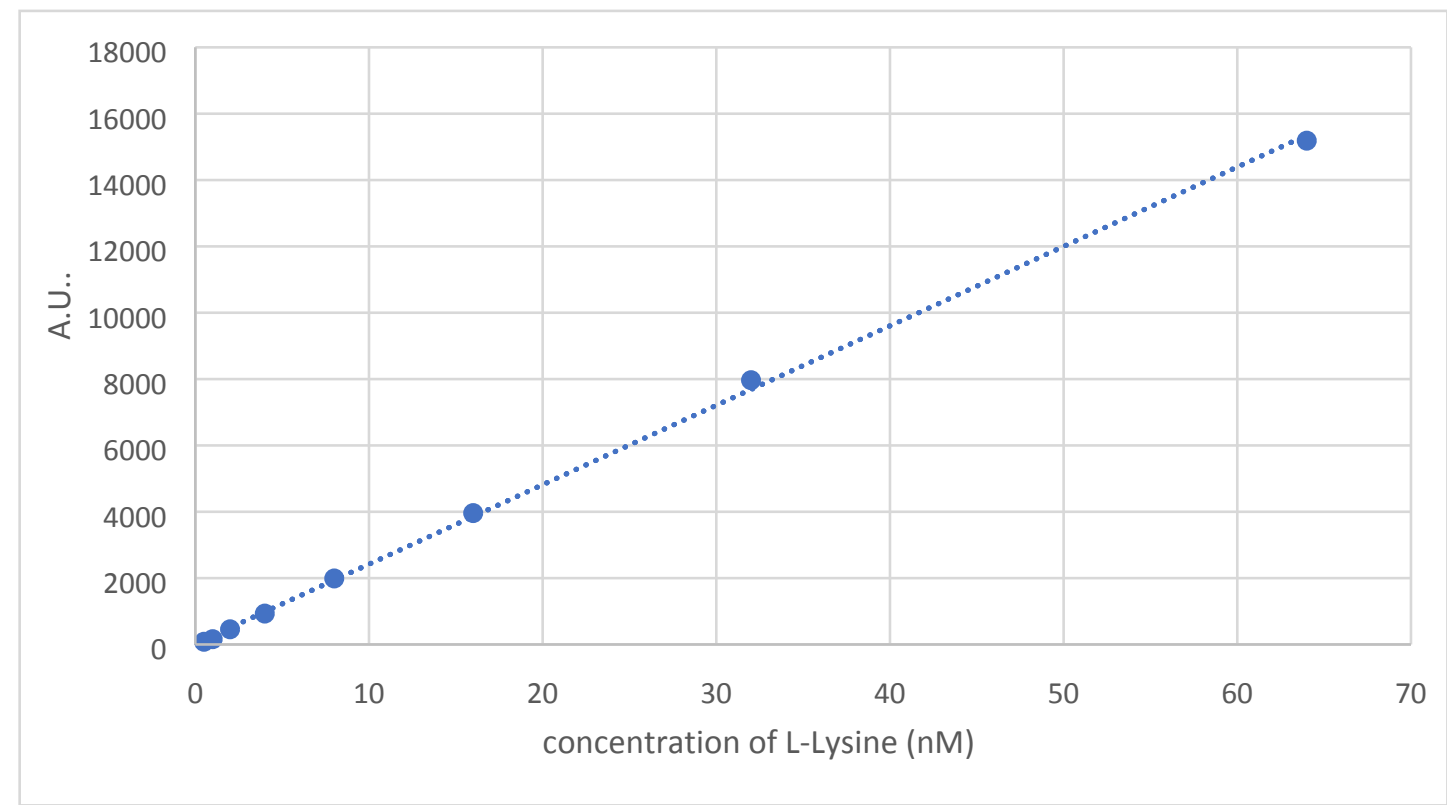

Figure S13: Standard curve of L-Lysine for quantification of LAP degradation 


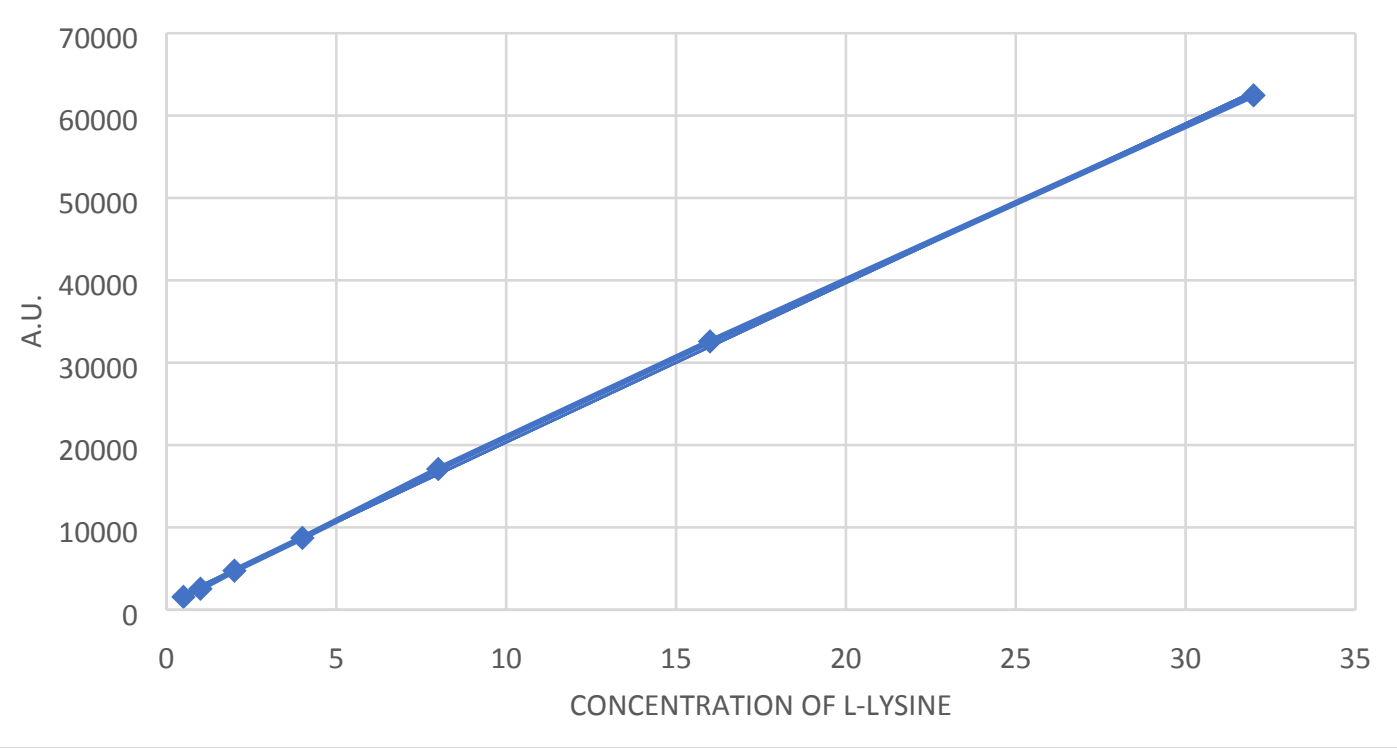

Figure S14: Standard curve of L-Lysine for quantification of trypsin degradation
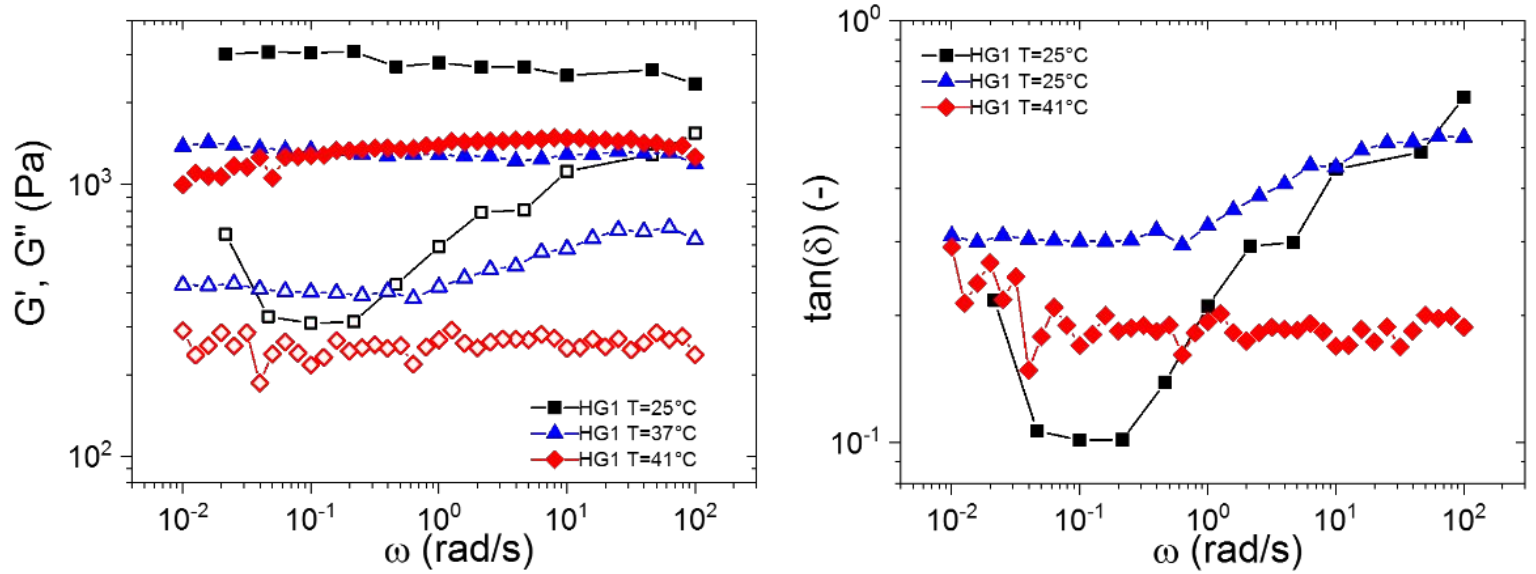

Figure S15: Dynamic frequency sweep in terms of A) storage modulus $\mathrm{G}^{\prime}$ (solid symbols) and loss modulus $\mathrm{G}^{\prime \prime}$ (open symbols), and B) the loss factor $\tan (\delta)$ for the hydrogel $\mathrm{HG} 1$ at $25^{\circ} \mathrm{C}$ (squares), $37^{\circ} \mathrm{C}$ (triangles) and $41^{\circ} \mathrm{C}$ (diamonds), $\mathrm{pH}=7.4 \mathrm{Lines}$ are a guide for the eye. The scattering of the data at $41{ }^{\circ} \mathrm{C}$ is due to the torque signal being close to the minimum resolution of the instrument. Other issues such as slight increase of $G^{\prime}$ at low frequencies due to equilibration issues, as discussed in the manuscript. In all rheological figures the size of the symbols is at or exceeds the size of error bars (see manuscript). 


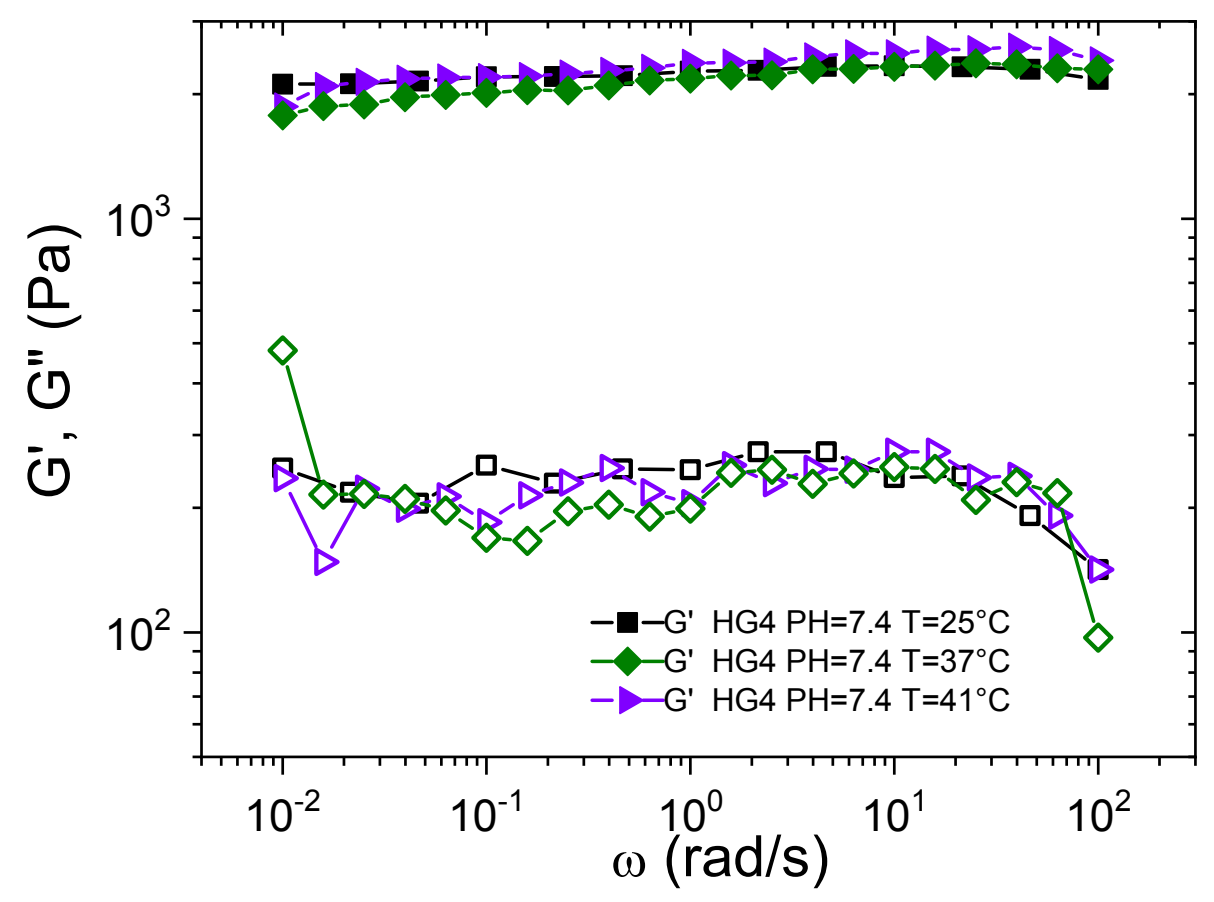

Figure S16: Dynamic frequency sweep in terms of storage modulus $\mathrm{G}^{\prime}$ (solid symbol) and loss modulus $\mathrm{G}^{\prime \prime}\left(\right.$ open symbol) for the hydrogel $\mathrm{HG} 4$ with $\mathrm{pH}=7.4$ at $25{ }^{\circ} \mathrm{C}$ (squares), $37{ }^{\circ} \mathrm{C}$ (triangles), and $41{ }^{\circ} \mathrm{C}$ (diamonds). The strain amplitude was fixed to $0.1 \%$ for all temperatures. Solid lines are a guide for the eye.
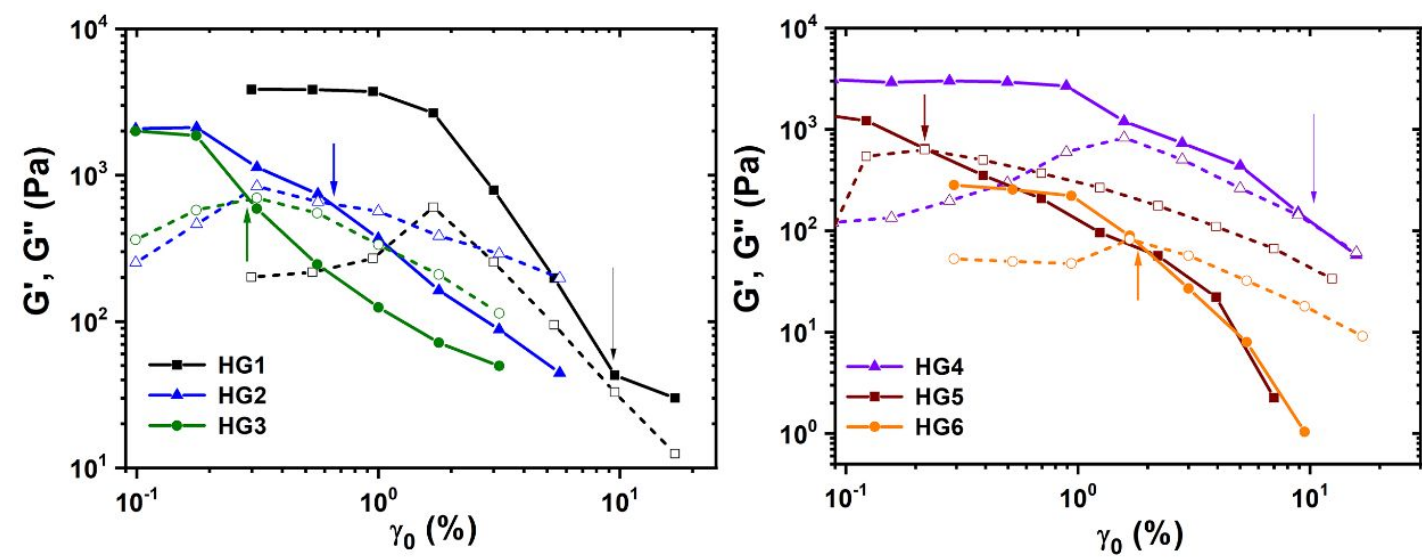

Figure S17: Dynamic strain sweeps in terms of storage modulus G' (solid symbols) and loss modulus G" (open symbols) as a function of strain amplitude $\gamma_{0}$ at the angular frequency $\omega=1 \mathrm{rad} / \mathrm{s}$ for the hydrogels (A) HG1 (squares), HG2 (triangles) and HG3 (diamonds) with the same polypeptide molecular characteristics and different PEO molecular weight. (B) HG4 (squares), HG5 (triangles) and HG6 (diamonds) with the 
same PEO molecular weight and different polypeptide molecular characteristics. The measurements were performed at $25^{\circ} \mathrm{C}, \mathrm{pH}=7.4$, and frequency equal to $1 \mathrm{rad} / \mathrm{s}$. The arrows the crossover points (for HG1 there is no real crossover that the highest strain amplitude data are uncertain, so the arrow is our expectation based on the overall rheological response). Lines are drawn to guide the eye.

\section{REFERENCES}

1. Hadjichristidis, N.; Iatrou, H.; Pispas, S.; Pitsikalis, M., Anionic polymerization: High vacuum techniques. Journal of Polymer Science Part aPolymer Chemistry 2000, 38 (18), 3211-3234.

2. Iatrou, H.; Frielinghaus, H.; Hanski, S.; Ferderigos, N.; Ruokolainen, J.; Ikkala, O.; Richter, D.; Mays, J.; Hadjichristidis, N., Architecturally induced multiresponsive vesicles from well-defined polypeptides. Formation of gene vehicles. Biomacromolecules 2007, 8 (7), 2173-2181.

3. Aliferis, T.; Iatrou, H.; Hadjichristidis, N., Living polypeptides. Biomacromolecules 2004, 5 (5), 1653-1656.

4. Mavrogiorgis, D.; Bilalis, P.; Karatzas, A.; Skoulas, D.; Fotinogiannopoulou, G.; Iatrou, H. Controlled polymerization of histidine and synthesis of welldefined stimuli responsive polymers. Elucidation of the structure-aggregation relationship of this highly multifunctional material. Polymer Chemistry 2014, 5, 6256-6278.

5. Heinz Maier-Leibnitz Zentrum et al..; KWS-1: Small-angle scattering diffractometer. Journal of large-scale research facilities, 2015, 1, A28.

6. Wignall, G.; D.; Bates, F. S., Absolute calibration of small-angle neutron scattering data. Journal of Applied Crystallography 1987, 20 (1), 28-40. 